\title{
Relationship between aggregation of rewards and the possibility of polymorphism in continuous snowdrift games.
}

\section{AUTHOR(S):}

Ito, Koichi; Ohtsuki, Hisashi; Yamauchi, Atsushi

\section{CITATION:}

Ito, Koichi ...[et al]. Relationship between aggregation of rewards and the possibility of polymorphism in continuous snowdrift games.. Journal of theoretical biology 2015, 372 : 47-53

\section{ISSUE DATE:}

2015-02-26

URL:

http://hdl.handle.net/2433/198323

\section{RIGHT:}

(c) 2015 Elsevier. Licensed under the Creative Commons Attribution-NonCommercial-NoDerivatives 4.0 International http://creativecommons.org/licenses/by-nc-nd/4.0/. NOTICE: this is the author's version of a work that was accepted for publication in Journal of Theoretical Biology. Changes resulting from the publishing process, such as peer review, editing, corrections, structural formatting, and other quality control mechanisms may not be reflected in this

document. Changes may have been made to this work since it was submitted for publication. A definitive version was

subsequently published in Journal of Theoretical Biology, Volume 372, Pages 47-53, doi:10.1016/J.jtbi.2015.02.015; ; 許諾 条件により本文ファイルは2016-02-26に公開.; This is not the published version. Please cite only the published version.;

この論文は出版社版でありません。引用の際には出版社版をご確認ざ利用ください。 

polymorphism in continuous snowdrift games

${ }^{1}$ Center for Ecological Research, Kyoto University, Hirano 2-509-3, Otsu 520-2113, Japan

${ }^{2}$ Department of Evolutionary Studies of Biosystems, School of Advanced Sciences, The Graduate University for Advanced Studies (SOKENDAI), Shonan village, Hayama, Kanagawa 240-0193, Japan

(Corresponding author)

Koichi Ito 
30

\section{Abstract}

The existence of intra-population variations in cooperation level has often been reported by some empirical studies. Evolutionary conditions of polymorphism in cooperation have been investigated by using a framework of the continuous snowdrift game. However, our insights from this framework have been limited because of an assumption that the cooperative reward is a function of total amount of investments within an interacting group. In many cases, payoffs may actually depend on the interactions between the effects of such investments, such as members share the sum of beneficial effects that are individually produced from their own investments. Alternatively, payoffs may depend multiplicatively on investment, such as when investments are complementary. In the present paper, we investigated the influence of such difference on the evolution of cooperation with respect to three aspects of the aggregating process of individuals' contributions for reward, i.e. (i) additive or multiplicative, (ii) aggregation of either investments or effects, and (iii) promotion of advantage or suppression of disadvantage. We analytically show that the possibilities of the emergence of polymorphism are different depending on the type of aggregation process classified from these three aspects. Polymorphism of cooperation level never emerges unless the aggregation process is the aggregation of investment or the multiplicative aggregation of effect with suppression of disadvantage. Our results show the necessary condition for the emergence of polymorphic cooperation levels that are observed in various taxonomic groups. [230 words]

\section{Keywords}

3 Adaptive dynamics, variance in cooperation, common good 
55

56

57

\section{Introduction}

Cooperative relationships have been widely observed in various taxonomic groups, involving bacteria, reptiles, mammals, and plants (Dugatkin 1997, Sachs et al. 2004, Melis and Semmann 2010, Raihani et al. 2012). Previous empirical studies about cooperation have often reported the existence of intra-population variation in cooperation level. For example, yeasts cooperate with neighbouring cells by sharing their profit in the process of resource decomposition, in which morphs with different levels of enzyme production can coexist (Greig and Travisano 2004). Animals or birds form groups and cooperate in being vigilant to approaching predators, but some individuals vary in their contributions to group vigilance (kangaroos, Carter et al. 2009; hyenas, Pangle and Holekamp 2010). In plants, it has been reported that anti-herbivore defence by an individual plant often reduces herbivory on its neighbouring ones (so-called “associational resistance”), but polymorphism of defence level is also observed in some cases (Agrawal et al. 2002, Hare and Elle 2002).

In general, selfish individuals will obtain a higher payoff than cooperative ones because they receive the benefits of cooperation without paying cooperative costs. Therefore, explaining the reason why cooperative individuals can persist in the presence of selfish ones is a challenging and important subject in evolutionary ecology. In order to solve this problem, some mechanisms have been proposed, which include kin selection (Hamilton 1964, 1972), future benefits (Clutton-Brock 2002) and frequency dependent selection for the cooperative traits in the context of game theory (Maynard Smith 1982). In particular, because game theory is a useful tool for describing the selection for the traits related to social interactions, game theory has been used for investigating the evolution of cooperation.

One important framework in game theory is the continuous snowdrift game, which 
80 is defined by Doebeli et al. (2004) as a game in which investment is a continuous

81 variable and "investment incur costs to the donor and accrue benefits to both the donor and the recipient." This differs from the more well-known continuous prisoner's dilemma game in which the investment does not yield a reward directly to the investor

84 (Killingback et al. 1999, Doebeli and Hauert 2005). It should be noted that some studies 85 of public goods game (Janssen and Goldstone 2006, Deng and Chu 2011, Chen et al. 2012) also satisfied the condition that was proposed by Doebeli et al. (2004), which can 87 be categorized into continuous snowdrift game.

Previous studies have indicated that under some conditions the continuous snowdrift game can predict evolutionary branching, and therefore dimorphism of 90 cooperation levels (Doebeli et al., 2004). Fluctuation in group size either stabilizes or destabilizes the dimorphism of cooperative levels depending on the shape of payoff function (Brännström et al. 2011), the existence of metapopulation structure relaxes the condition for the emergence of polymorphism (Parvinen 2011), and a small population

94 size prevents evolutionary branching (Wakano and Iwasa 2013). Functional forms of 95 reward and cost are also proposed as an important factor for the coexistence of 96 polymorphic cooperation levels (Archetti and Scheuring 2012). Doebeli et al. (2004)

97 indicated that the concavity of both reward and cost functions is a necessary and sufficient condition for the occurrence of evolutionary branching.

These previous studies, however, generally have paid less attention to the process

100 by which rewards result from investment. In the process of producing reward on 101 cooperation, each individual provides 'investment' in order to obtain some 102 advantageous 'effect,' the amount of which can be represented by a function of 103 investment (e.g. an investment $z$ produces an effect $f(z)$ ). For example, yeast produce 
104 enzymes in order to produce decomposition products, or in the group vigilance

105 individuals consume time in vigilance in order to detect their predators. In cooperative

106 interactions among multiple individuals, the contributions of neighbouring individuals

107 are aggregated, and consequently each individual obtains a resultant effect as the reward

108 of cooperation. We can consider some types of aggregation process depending on the

109 mechanism of the aggregation.

110 First, we can consider two aggregation stages depending on whether the individuals'

111 contributions are aggregated at investments or at effects that is produced by such

112 investment. These will only be equivalent if the aggregation is additive and the

113 relationship between investment and effects are linear. However, this is likely to be

114 unrealistic in most cases. For example, the amount of enzyme produced by yeast will

115 not be linearly related the obtained decomposition, because the decomposition rate

116 generally follow Michaelis-Menten kinetics (Zaks and Klibanov 1985). The detail of the

117 cooperation of yeast should be investigated by considering the chemical

118 reaction-diffusion process (e.g. Borenstein et al. 2013, Archetti 2014, Scheuring 2014).

119 However, for generality of analysis, we summarize those processes into two simple

120 equations, which are ineffective for quantitative predictions but effective for

121 investigation of essential mechanisms in the considered system. One is that each

122 individual produces enzyme $z$, and the total of this enzyme by all group mates, $\Sigma z$, is

123 used to produce decomposed products $f(\Sigma z)$, in which aggregation occur before

124 producing products. Alternatively, each individual invests energy $z$ to produce

125 decomposed products $f(z)$, the total of which, $\Sigma f(z)$, benefits the focal individual. In this

126 case, the aggregation occurs after the producing products. We call the former

127 “aggregation of investments” and the latter "aggregation of effects," respectively 
128 throughout the paper.

129 The second issue is how the factors are associated, i.e., "additive aggregation” or

130 “multiplicative aggregation” (e.g. $\Sigma z$ or $\Pi z)$. An additive aggregation often applies to

131 material benefits such as enzyme or decomposition products in yeast, but the

132 multiplicative aggregation is also conceivable. Consider group vigilance: if individual

133 bouts of vigilance overlap, the probability of spotting a predator is calculated by the

134 product of the probabilities of a single individual not finding an enemy. This is also a

135 greatly simplified situation, and the group vigilance should be investigated by

136 considering behavioural process in detail (e.g. Proctor et al. 2002). However, this

137 example shows that multiplicative benefit is more appropriate in some cases. Moreover,

138 we can also consider the difference of reward type, i.e. the reward is obtained through

139 whether promotion of advantage or suppression of disadvantage. In the cooperation in

140 yeast, more investments promote the advantage by producing more decomposition

141 products. Contrarily, in group vigilance, more investments suppress the disadvantage by

142 reducing the risk of predator attack. This difference will appear as whether the reward

143 term is positive and $f(z)$ is increasing, or the reward term is negative and $f(z)$ is a

144 decreasing function.

145 Accordingly, we can categorize the aggregation processes of producing reward with

146 respect to three aspects, i.e. (i) additive or multiplicative aggregation, (ii) aggregation of

147 investments or effects, and (iii) promotion of advantage or suppression of disadvantage

148 (Figure 1). As a component of the payoff function, previous studies are mainly focused

149 on the functional shapes of reward and cost, but not considered effects of aggregation

150 process. For example, most of the previous models of continuous snowdrift game

151 assumed the aggregation process, which we call "additive aggregation of investments 
152 with promotion of advantage." But in order to understand general properties of

153 evolutionary processes in a continuous snowdrift game, we should analyse its

154 evolutionary dynamics for various possible aggregation processes in rewards.

155 In this analysis, we investigate two properties: (i) the possibility of the occurrence

156 of evolutionary branching leading to polymorphism and (ii) the possibility of the

157 sustained coexistence of polymorphism. We also compare the influence of the

158 functional shapes of effect and cost among aggregation processes. We analytically show

159 that these possibilities are different depending on the type of aggregation process of

160 rewards. Our results show us the condition for the emergence of polymorphisms that are

161 observed in various organisms.

162

163

\section{Model}

\subsection{Payoff function}

165

We consider a sufficiently large asexual population, individuals of which are categorized into morphs based on their trait values. For social interactions, it is assumed that $N$ individuals are randomly chosen from this population to form a group, within which members interact with each other. Consider a certain individual in an interacting group, a trait value of which is represented by $y$. The payoff of this individual depends on the traits of its $N-1$ group mates. Those group mates can be polymorphic in trait

171 values. In particular, we refer to the trait value and number of $i$-th morph in the group 172 except for the focal individual as $x_{i}$ and $n_{i}\left(n_{i} \geq 0\right.$ and $\left.\Sigma n_{i}=N-1\right)$, respectively. When the

173 total number of morphs in the population is $m$, an assemblage of group mates is 174 represented by $\left\{\left(x_{i}, n_{i}\right)\right\}_{\{i=1 \ldots m\}}$, which is an assemblage of $\left(x_{i}, n_{i}\right)$ of all morphs. In this 175 group, the payoff of the individual with trait $y$ given the traits of the other group 176 members is 


$$
w\left(y \mid\left\{\left(x_{i}, n_{i}\right)\right\}_{i=1 \ldots m}\right)=F\left(y \mid\left\{\left(x_{i}, n_{i}\right)\right\}_{i=1 \ldots m}\right)-g(y),
$$

where the first and second terms of the right-hand side are the reward and the cost of is a monotonically increasing function of $y$.

181 Because the group mates are randomly chosen from the population, the expected 182 payoff of the focal individual with trait $y$ is calculated by asking the expectation over all possible combinations of group mates. Let us represent the frequency of $i$-th morphs in the population as $p_{i}\left(\Sigma p_{i}=1\right)$. The probability of obtaining the composition of group article as

$$
\left(\begin{array}{c}
N-1 \\
\mathbf{n}
\end{array}\right) \mathbf{p}^{\mathbf{n}}=\frac{(N-1) !}{n_{1} ! n_{2} ! \cdots n_{m} !} p_{1}^{n_{1}} p_{2}{ }^{n_{2}} \cdots p_{m}{ }^{n_{m}}
$$

Averaging Eq.(1) with this probability, the expected payoff of the focal individual with phenotypic value $y$ is

$$
W\left(y \mid\left\{\left(x_{i}, p_{i}\right)\right\}_{i=1 \cdots m}\right)=\sum_{\mathbf{n}}\left[\left(\begin{array}{c}
N-1 \\
\mathbf{n}
\end{array}\right) \mathbf{p}^{\mathbf{n}} F\left(y \mid\left\{\left(x_{i}, n_{i}\right)\right\}_{i=1 \cdots m}\right)\right]-g(y) .
$$

\subsection{Five possible aggregation processes}

In this study, we consider the difference of the aggregation process of individual contributions on the reward. For simplicity of explanation, we represent the trait values of all group members by $\left(z_{1}, z_{2}, \ldots, z_{N}\right)$, including the focal individual's. Each individual provides investment, $z_{i}$, which results in some advantageous effect. The individual investments are translated to the reward differently depending on its aggregation process as illustrated in Figure 1. Thus, the reward function of focal individual, $F$, is 
200 functional shape of effect $f(z)$ determined by investment $z$. We assume that the reward $201 F(\bullet)$ always increases as the amount of investments increases, although $f(\bullet)$ can be both 202 increasing and decreasing functions depending on the aggregation process. We represent 203 the effect functions in former and latter case as $f_{+}(\bullet)$ and $f(\cdot)$, respectively.

204 We consider aggregation processes with respect to three aspects, i.e. (i) additive or 205 multiplicative, (ii) aggregation of either investments or effects, and (iii) promotion of 206 advantage or suppression of disadvantage. By considering the every combination of 207 these aspects, we obtain eight types of aggregation process. However, expect for the combination of multiplicative aggregation of effect, aspect (iii) does not yield an 209 essential difference, because one can always convert a monotonically decreasing 210 function $f_{-}(\cdot)$ to a monotonically increasing function $f_{+}(\cdot)$ simply by adding a minus sign 211 in its front. On the other hand, in multiplicative aggregation of effects, where the 212 "promotion of advantage"-type reward function, $\Pi f_{+}\left(z_{j}\right)$, cannot be transformed to the 213 “suppression of disadvantage”-type reward function, - $\Pi f_{-}\left(\mathrm{z}_{j}\right)$. This difference may seem

214 trivial. However, the functional form of aggregation process influences the curvature of

215 the reward function (and then payoff function), which can be a significant determinant 216 of evolutionary dynamics. It may ultimately affect the possibility of the occurrence of 217 polymorphism in cooperation level.

218 As a result, we have five types of aggregation process (Figure 1). The first four 219 functions, $f(\bullet)$ and $f_{+}(\bullet)$, are monotonically increasing. The bottom function, $f(\cdot)$, is 220 monotonically decreasing. We also assume that $z$ is always positive in multiplicative 221 aggregation of investments, and that $f_{+}$and $f$ - are positive in multiplicative aggregation of effects. 
224

225

226

227

228

229

230

231

232

233

234

235

236

237

238

239

240

241

$$
\frac{d x_{i}}{d t}=\left.\beta_{i}\left(x_{1}, x_{2}, \cdots x_{m}\right) \frac{\partial W\left(y \mid x_{1}, x_{2}, \cdots x_{m}\right)}{\partial y}\right|_{y=x_{i}},
$$

where $\beta_{i}\left(x_{1}, x_{2}, \ldots x_{m}\right)$ is a positive coefficient determining the rate of evolutionary change of $x_{i}$. Therefore, the condition for the evolutionary singular coalition $x_{i=1 \ldots m}^{*}$ (or the evolutionary singular point for $m=1$ ) can be written as

$$
\left.\frac{\partial}{\partial y} W\left(y \mid x_{1}^{*}, x_{2}^{*}, \cdots x_{m}^{*}\right)\right|_{y=x_{j}^{*}}=0 \quad \forall j=1 \cdots m .
$$


stabilities of the solution concerning their evolutionary property, i.e. convergence stability (CS) and evolutionary stability (ES). If the solution is both CS and ES, the solution is a continuous stable state (CSS: Eshel 1983). If the singular solution is CS but not ES, an evolutionary branching occurs and a new morph with a different cooperation

250 level joins the population. If the singular solution is not CS, such a solution will never

251 be reachable as a result of evolution. According to Geritz et al. (1998), the solution is 252 ES when

$$
\frac{\partial^{2}}{\partial y^{2}} W\left(y\left|x_{1}^{*}, x_{2}^{*}, \cdots x_{m}^{*}\right|_{y=x_{j}^{*}}<0 \quad \forall j=1 \cdots m\right.
$$

254 255 256

is satisfied. The condition of CS can be investigated by examining a matrix $\mathbf{M}$ whose ( $i$, j)-element is

$$
\mathbf{M}_{i j}=\frac{\partial}{\partial x_{j}}\left(\left.\beta_{i}\left(x_{1}^{*}, x_{2}^{*}, \cdots x_{m}^{*}\right) \frac{\partial W\left(y \mid x_{1}^{*}, x_{2}^{*}, \cdots x_{m}^{*}\right)}{\partial y}\right|_{y=x_{i}^{*}}\right) \text {. }
$$

The solution is CS when real parts of all the eigenvalues of $\mathbf{M}$ are negative (Leimar 2009).

\subsection{Evolutionary polymorphism and sustained polymorphism}

By examining these evolutionary properties, we investigate two types of possibilities of the existence of polymorphism. First, we consider the possibility of emergence of polymorphism by successive evolutionary branching from a monomorphic state. If there is a feasible singular coalition in a population with $m=l$ morphs and an evolutionary branching is possible at the singular coalition, an evolutionary branching occurs and the number of morphs becomes $m=l+1$. By successively examining this condition from a monomorphic to polymorphic population, we investigate how many morphs can potentially appear through evolutionary 
269 branching. In the present study, we refer to this potential as the possibility of 270 “evolutionary polymorphism.” Notice that the word "evolutionary” is a key here,

271 because this term suggests the possibility of polymorphism through an evolutionary 272 process. In summary, we say that an evolutionary polymorphism with $l$ morphs is 273 possible if (i) the singular coalitions can be CS but non-ES for states with $1,2, \ldots, l-1$ 274 morphs, (ii) singular coalitions can be feasible for states with $1,2, \ldots, l$ morphs, and 275 (iii) the singular coalition can be CS and ES for a state with $l$ morphs. polymorphism for five types of aggregation process. Although the detail of the analyses are shown in Appendix A, here we show the analyses for additive aggregation of effects (AE) and additive aggregation of investment (AI) as the examples. In AE type, the payoff of a mutant with $y$ in a monomorphic population with $x$ can be written as

$$
W(y \mid x)=(N-1) f(x)+f(y)-g(y) \text {. }
$$

According to Eqs. (7) and (8), the singular points $x^{*}$ become CS but non-ES when

$$
f^{\prime}\left(x^{*}\right)<g^{\prime \prime}\left(x^{*}\right)<f^{\prime \prime}\left(x^{*}\right) \text {. }
$$

284 Since left and right inequalities are never satisfied simultaneously, an evolutionary 285 branching never occurs. Therefore, the condition (i) is never satisfied when $l>1$, and 286 we can conclude that the evolutionary polymorphism with two or more morphs is impossible in AE type. On the other hand, in AI type, the payoff function can be written as

$$
W(y \mid x)=f(a(N-1) x+y)-g(y)
$$

290 The singular points $x^{*}$ become CS but non-ES in a monomorphic population when

$$
N f^{\prime \prime}\left(N x^{*}\right)<g^{\prime \prime}\left(x^{*}\right)<f^{\prime \prime}\left(N x^{*}\right) .
$$

292 This condition can be satisfied if $g\left(x^{*}\right)<f\left(N x^{*}\right)<0$, therefore we cannon reject the 
possibility that the condition (i) with $l=2$ can be satisfied. Moreover, we can show that

294 we cannot also reject the possibility of the existence of a solution satisfying the conditions of evolutionary polymorphism with $l \geq 2$ (Appendix A). Consequently, in AI type, the evolutionary polymorphism with two or more morphs is potentially possible. We investigated the possibility of evolutionary polymorphism for the other types of aggregation process (MI, pME and sME) by using similar analysis (see Appendix A for the detail of the analysis).

Second, we consider the possibility of the sustained coexistence of multiple morphs in a polymorphic population. Even if we find the number of morphs $k(<l)$ such that a 302 singular coalition with $k$ morphs never satisfies a branching condition (hence the

303 emergence of polymorphism with $l$ morphs is never possible through a successive evolutionary branching), one cannot reject the possibility of a sustained polymorphic solution when the polymorphism is already established for some historical reason. For example, when individuals immigrate from different environments, or when mutations with large effects on trait values occur, the population can be polymorphic potentially without the occurrence of evolutionary branching. Such a potential can simply be examined by studying conditions for both feasibility and evolutionary stability of an evolutionarily singular coalition with $l$ morphs, ignoring the property of a singular

311 solution with $1,2, \ldots, l-1$ morphs (see Appendix A). In the present study, we refer to this 312 potential as the possibility of "sustained polymorphism." Note that the word "sustained"

313 is a key here, because we do not a priori assume any mechanisms of how a polymorphic 314 population with $l$ morphs was initially built up. To summarize, we say that a sustained polymorphism with $l$ morphs is possible if (i) a singular coalition can be feasible for 
the evolutionary polymorphism with $l$ morphs is possible, it automatically suggests that the sustained polymorphism with $l$ morphs is also possible.

Here we show the examples of the analysis for the possibility of the sustained polymorphism for AE type and AI type. Please see Appendix A for more details and the analyses for the other types of aggregation. In AE type, the necessary condition for the coexistence of $m$ morphs is that the simultaneous equations

$$
\begin{aligned}
& f\left(x_{i}^{*}\right)-g\left(x_{i}^{*}\right)=f\left(x_{j}^{*}\right)-g\left(x_{j}^{*}\right) \quad \forall \quad i, j=1 \ldots m, \\
& f^{\prime}\left(x_{i}^{*}\right)=g^{\prime}\left(x_{i}^{*}\right) \quad \forall \quad i=1 \ldots m,
\end{aligned}
$$

have a solution. Because Eqs. (13a) and (13b) yield $m-1$ and $m$ constraints respectively, there are $2 m-1$ constraints in total. The number of unknown variables in Eqs. (13) is, $m$ (i.e., trait value $x^{*}{ }_{i=1 \ldots m}$ ). Since the number of variables is fewer than that of constraints for $m>1$, two or more morphs cannot coexist; the sustained polymorphism with two or more morphs is impossible in AE. On the other hand, in AI type, we already know that evolutionary branching is possible (see Eq. (12) and Appendix A). Since the evolutionary polymorphism is a sufficient condition for the sustained polymorphism as mentioned above, the sustained polymorphism with two or more morphs is also possible in this case. By using similar analysis, we investigated the sustained polymorphism for the other types of aggregation process (i.e. MI, pME and sME, see Appendix A).

It should be noticed that we focused on necessary conditions for evolutionary polymorphism and sustained polymorphism rather than sufficient conditions. These conditions do not ensure that a polymorphism with an appropriate number of morphs always occurs. However, it is surely ensured that when the concerning conditions are violated those phenomena never occur. Our study is thus useful in elucidating evolutionary conditions for polymorphism, and it has direct implication to empirical 
studies.

\section{Results}

\subsection{Influence of the aggregation process}

We analytically investigate the possibilities of evolutionary and sustained

polymorphism by applying the general approach described above for five types of aggregation process (see Appendix A for details of our analyses) under the condition without any restriction for the functional shapes of the effect $f(\bullet)$ and the cost $g(\bullet)$. Table 1 summarizes the result of the analytical investigation. According to the present analysis, both $\mathrm{AI}$ and MI could result in evolutionary and sustained polymorphism with more than two morphs. Even in those cases, the polymorphism with more than two morphs tends to occur under restricted conditions only. Carefully choosing adequate functional forms and parameters, we can show the emergence of polymorphism through an adaptive dynamic process by using individual-based simulations (Figure 2, the detail of the simulation is written in Appendix B). On the other hand, AE leads to monomorphism only, resulting in neither dimorphism nor polymorphism. This is because the singular solution does not depend on the amount of investments by the other individuals (see Eq. (A9) and (A10) in Appendix A). This implies that interactions among individuals are not relevant but that the efficiency of one's contribution is simply maximized through an evolutionary process. Therefore as a solution of simple optimization it results in a monomorphic state.

362 Interestingly, in the case of ME, the property is different between whether cooperation is advantage-promoting or it is disadvantage-suppressing. In the sME, monomorphism and dimorphism are possible although polymorphism with more than 
hand, for the pME, dimorphism never results from an evolutionary branching, but is sustained. This implies that in such a case, mutations with large effects or migrations from another population are needed to result in dimorphism. We also consider the situation that an individual investment either more or less influences its own reward than those from other members. However, such inequality of the group member's contribution does not alter the general results of our analysis (see Appendix A).

\subsection{Influence of the functional shapes of effect and cost}

The functional shapes also influence the possibility of the evolutionary branching.

Next, we examine the influence of their functional shapes on the evolutionary process. To do so, we categorize the functional shapes simply into four types, i.e. linear, convex, concave, and the other functional shapes (e.g. sigmoid), we call the last type as complex type. We examine the condition for evolutionary branching by focusing on AI, MI and sME (see Appendix A) and reveal the combination of the functional shapes of effect and cost that realizes evolutionary branching. Similarly to the above analyses of evolutionary and sustained polymorphism, we consider necessary conditions under which polymorphism occurs.

Figure 3 shows the summary of the analysis. In both AI and MI, evolutionary branching can occur only when both effect and cost functions have a decelerating shape at the singular solution. Therefore, both effect and cost functions must be in either a concave or complex shape for the occurrence of branching. On the other hand, in sME, evolutionary branching can occur when either effect or cost function has a decelerating shape at the singular solution, and, therefore, either effect or cost function should be either a concave or a complex type of function for evolutionary branching. 
391

392

393

394

395

396

397

398

399

400

401

402

403

404

405

406

407

408

409

410

411

412

413

414

415

\section{Discussion}

In the present study, we show that the aggregation process of rewards significantly influences the possibility of both evolutionary and sustained polymorphism in cooperation level. Previous studies have reported multiple factors affecting the emergence of polymorphism in cooperation levels (Doebeli et al. 2004, Brännström et al. 2011, Parvinen 2011, Wakano and Iwasa 2013). However, they focussed on the payoff functions that conditionally enabled polymorphic cooperation level to evolve. Although some previous studies investigated the influence of the aggregation process of reward with focusing on some specific cases (e.g. reaction-diffusion process of yeast in Borenstein et al. 2013, Archetti 2014, Scheuring 2014), the general pattern of the influence of the aggregation process on the evolution of cooperation has not been revealed. The present analysis significantly generalised these works to show the potential possibility of evolution of polymorphism for a wider class of payoff functions with various aggregation processes of reward effects. We indicated that polymorphism of cooperation levels never emerges from the evolutionary process unless the aggregation process is $\mathrm{AI}$, MI or sME type. Consequently, we show that the type of aggregation process is an important and remarkable element of cooperation when we consider the variation of cooperation levels.

By applying our findings to the empirical examples of cooperation, we can predict the possibility of variation in cooperation levels from the information of aggregation process, or suggest the mechanism of aggregation process itself under an existence of variation of cooperation level. In the cooperation of yeasts, for example, both $\mathrm{AI}$ and $\mathrm{AE}$ types are possible depending on whether they share the decomposing enzyme or the decomposed products. According to our results, cooperation level becomes always monomorphic under AE, but can be polymorphic under AI (see Table 1). In reality, 
416 yeasts may share both enzyme and decomposed products probably through the chemical

417 reaction-diffusion process, and our classification of the type of aggregation in yeast is

418 generally difficult to apply to realistic situations directly. However, it is possible to

419 predict from our analysis that some level of enzyme sharing with neighbours is

420 necessary for the coexistence of multiple morphs with different abilities of enzyme

421 production (Greig and Travisano 2004). In addition, we can also predict that both the

422 amount of decomposed products and the individual cost of producing enzyme should be

423 concave functions of amounts of the total enzyme and the individual enzyme,

424 respectively (see Table 2). This prediction is supported by an experimental study (Gore 425 et al. 2009).

426 Polymorphism in the plant defence (Agrawal et al. 2002, Hare and Elle 2002) can 427 also be caused by the cooperative interaction called associational resistance, but the 428 aggregation process of them depends on the mechanism of the associational resistance. In order to discuss the effect of the aggregation process in the plant defence, let us 430 consider some simplified situations. For example, one possible situation is the 431 production of toxic chemicals against herbivores which visit plant individuals one by one and feed on them (e.g. grasshoppers or caterpillars). Because toxic chemicals will reduce the activity or survival probability of the feeding herbivores, the production of toxic chemicals will mitigate the herbivory pressure of neighbouring. If the toxic 435 chemicals accumulate in the feeding herbivore and eventually result in the reduction of 436 herbivores' survival probability, the aggregation process is categorized to AI. On the 437 other hand, if the toxic chemicals do not accumulate in the herbivores but reduce their 438 survival probability multiplicatively by each feeding event, the aggregation process will 
440 chemical production can emerge. We can also consider other type of associational 441 defence in plants that is against herbivores reproducing on plant individual (e.g. aphids, 442 spider mites or white flies). If the herbivores disperse to the neighbouring plant 443 individuals after the reproduction, the individual defence will reduce the number of 444 spreading herbivores. In this case, the number of herbivores on each plant will be a 445 summation of remaining herbivores and dispersal from neighbours, the aggregation 446 process of which will be categorized to $\mathrm{AE}$, always resulting in monomorphism of 447 defence.

448 In the analysis of the possibility of evolutionary and sustained polymorphism, we assume that the cooperative trait is determined genetically and that it evolves with small 450 mutations. However, in the cooperation based on the flexible decision-making and behavioural action (e.g. group vigilance against enemy), individuals can change their cooperation level discontinuously at any time. In such a case, coexistence of multiple phenotypes can be realized by a mixed strategy with multiple tactics rather than

454 phenotypic polymorphism; hence we cannot apply the presented analysis directly to 455 such behavioural cooperation. However, by an adequate extension of the present 456 analysis, the conditions for sustained polymorphism are applicable to behavioural 457 polymorphism that is controlled by flexible decision-making by individuals (Appendix 458 C). Consequently, we can discuss behavioural cooperation based on the presented 459 results. For example, in the group vigilance for natural enemy, individuals seem to 460 aggregate the probabilities of finding enemies rather than the investment in the vigilance 461 itself, which would correspond to the aggregation of effects. When every group member 462 scans the same area, the probability of no one finding an enemy is the product of the 463 probabilities that each individual fails to find it, which can be categorized to sME. On 
464 the other hand, when each individual scans a different area, the probability of finding an 465 enemy will be a summation of the probabilities of each finding an enemy, which coincides with AE. Therefore, we can predict that polymorphisms of vigilance level (Carter et al. 2009, Pangle and Holekamp 2010) will be observed only in the former case. However, we should consider carefully when we apply the present result to the 469 behavioural polymorphisms. Generally speaking, behavioural polymorphism is realized 470 not only by a mixed strategy that is evolutionarily stable, but also by condition-dependent alternative strategies. None of our "impossibility" results rejects the possibility of behavioural polymorphism realized by condition-dependent alternative strategies.

In the present analysis, the payoff function is simply formulated as reward minus cost, but another expression may be possible depending on the mechanism of considering cooperation. When we consider the situation that individuals use a common-pool resource cooperatively, consuming the resource of an individual increases its own payoff but reduces the common rewards. In such a case, an individual's cost will be a function of both own and other's investments while its reward will be a function of only its own investment, e.g. $f(z)-g(\Sigma z)$ (Killingback et al. 2010). Nevertheless by applying a translation $\hat{z}=-z, \hat{f}(\bullet)=-g(\bullet)$ and $\hat{g}(\bullet)=-f(\bullet)$, we can apply our results to such case, the result of which is consistent with the original result of Killingback et al. (2010). A payoff can often be expressed as the product of reward and cost, (e.g. $f(\Sigma z) g(z)$ in Brännström and Dieckmann 2005), but we can simply map such cases to our framework by using the $\log$ translation of payoff (e.g. $\log [f(\Sigma z)]$ $\left.486 \log \left[g(z)^{-1}\right]\right)$. 
488 the evolution of polymorphic cooperation level, there are some open questions. In the

489 present study, we categorized the aggregation processes according to the stage of 490 aggregation, i.e. the aggregation of investments or effects. However, the simultaneous 491 aggregation of both investments and effects is also possible. In reality, such an 492 aggregation process can be considered, e.g. in the cooperation of yeast, they may share 493 both enzyme and decomposed products rather than either of those. Such a multi-stage 494 aggregation may alter the properties of the evolution of polymorphism. In addition, the 495 present analysis is based on asexual reproduction, ignoring exchanges of genetic 496 information between individuals. In order to understand observed polymorphism in 497 nature, we have to extend our approach to sexual reproduction. Moreover, the studies 498 about the aggregation process with focusing on more specific cooperative processes are 499 also important for detecting the biological factors or parameters which determine the 500 emergence of polymorphism. 
502 Acknowledgements

503 We thank A. D. Higginson for his helpful comments on the manuscript and the 504 members of the Centre for Ecological Research, Kyoto University, for their comments 505 and encouragement. We are greatly appreciated to two anonymous referees for their 506 helpful comments and suggestions. This research was supported financially by the 507 Global COE Program A06 at Kyoto University, and by the Core-to-Core project (20004) 508 and KAKENHI (11007744) from the Japan Society for the Promotion of Science (JSPS) 509 to AY, and by KAKENHI (25118006) to HO, and by Grant-in-Aid for JSPS Fellows $510 \quad(26 * 472)$ to KI. 
512

513

514

515

516

517

518

519

520

521

522

523

524

525

526

527

528

529

530

531

532

533

534

535

536

537

538

539

540

541

542

543

544

545

546

547

548

\section{References}

Agrawal, A.A., Conner, J.K., Johnson, M.T.J., Wallsgrove, R., 2002. Ecological genetics of an induced plant defense against herbivores: additive genetic variance and costs of phenotypic plasticity. Evolution 56, 2206-2213. doi:10.1111/j.0014-3820.2002.tb00145.x

Archetti, M., Scheuring, I., 2012. Game theory of public goods in one-shot social dilemmas without assortment. J. Theor. Biol. 299, 9-20.

$$
\text { doi:10.1016/j.jtbi.2011.06.018 }
$$

Archetti, M., 2014. Stable Heterogeneity for the Production of Diffusible Factors in Cell Populations. PLoS ONE 9, e108526. doi:10.1371/journal.pone.0108526

Bishop, D.T., Cannings, C., 1978. A generalized war of attrition. Journal of Theoretical Biology 70, 85-124. doi:10.1016/0022-5193(78)90304-1

Borenstein, D.B., Meir, Y., Shaevitz, J.W., Wingreen, N.S., 2013. Non-local interaction via diffusible resource prevents coexistence of cooperators and cheaters in a lattice model. PLoS ONE 8, e63304. doi:10.1371/journal.pone.0063304

Brännström, Å., Dieckmann, U., 2005. Evolutionary dynamics of altruism and cheating among social amoebas. Proc. R. Soc. B 272, 1609-1616.

doi:10.1098/rspb.2005.3116

Brännström, Å., Gross, T., Blasius, B., Dieckmann, U., 2011. Consequences of fluctuating group size for the evolution of cooperation. J. Math. Biol. 63, 263-281. doi:10.1007/s00285-010-0367-3

Carter, A.J., Pays, O., Goldizen, A.W., 2009. Individual variation in the relationship between vigilance and group size in eastern grey kangaroos. Behav Ecol Sociobiol 64, 237-245. doi:10.1007/s00265-009-0840-4

Chen, X., Szolnoki, A., Perc, M., Wang, L., 2012. Impact of generalized benefit functions on the evolution of cooperation in spatial public goods games with continuous strategies. Phys. Rev. E 85, 066133. doi:10.1103/PhysRevE.85.066133

Clutton-Brock, T., 2002. Breeding together: kin selection and mutualism in cooperative vertebrates. Science 296, 69-72. doi:10.1126/science.296.5565.69

Cressman, R., Hofbauer, J., 2005. Measure dynamics on a one-dimensional continuous trait space: theoretical foundations for adaptive dynamics. Theoretical Population Biology 67, 47-59. doi:10.1016/j.tpb.2004.08.001

Deng, K., Chu, T., 2011. Adaptive evolution of cooperation through Darwinian dynamics in public goods games. PLoS ONE 6, e25496. doi:10.1371/journal.pone.0025496

Dieckmann, U., Law, R., 1996. The dynamical theory of coevolution: a derivation from stochastic ecological processes. Journal of Mathematical Biology 34, 579-612. doi:10.1007/BF02409751 
549

550

551

552

553

554

555

556

557

558

559

560

561

562

563

564

565

566

567

568

569

570

571

572

573

574

575

576

577

578

579

580

581

582

583

584

585

Doebeli, M., Hauert, C., 2005. Models of cooperation based on the Prisoner's Dilemma and the Snowdrift game. Ecology Letters 8, 748-766. doi:10.1111/j.1461-0248.2005.00773.x

Doebeli, M., Hauert, C., Killingback, T., 2004. The evolutionary origin of cooperators and defectors. Science 306, 859-862. doi:10.1126/science.1101456

Dugatkin, L.A., 1997. Cooperation among animals: an evolutionary perspective. Oxford University Press.

Eshel, I., 1983. Evolutionary and continuous stability. Journal of Theoretical Biology 103, 99-111. doi:10.1016/0022-5193(83)90201-1

Geritz, S.A.H., Kisdi, E., Meszena, G., Metz, J.A.J., 1998. Evolutionarily singular strategies and the adaptive growth and branching of the evolutionary tree. Evolutionary Ecology 12, 35-57.

Gore, J., Youk, H., van Oudenaarden, A., 2009. Snowdrift game dynamics and facultative cheating in yeast. Nature 459, 253-256. doi:10.1038/nature07921

Greig, D., Travisano, M., 2004. The Prisoner’s Dilemma and polymorphism in yeast SUC genes. Proceedings of the Royal Society B: Biological Sciences 271, S25-S26. doi:10.1098/rsbl.2003.0083

Hamilton, W.D., 1964. The genetical evolution of social behaviour. Journal of Theoretical Biology 7, 1-52. doi:10.1016/0022-5193(64)90038-4

Hamilton, W.D., 1972. Altruism and related phenomena, mainly in social insects. Annual Review of Ecology and Systematics 3, 193-232. doi:10.1146/annurev.es.03.110172.001205

Hare, J.D., Elle, E., 2002. Variable impact of diverse insect herbivores on dimorphic datura wrightii. Ecology 83, 2711-2720. doi:10.1890/0012-9658(2002)083[2711:VIODIH]2.0.CO;2

Janssen, M.A., Goldstone, R.L., 2006. Dynamic-persistence of cooperation in public good games when group size is dynamic. Journal of Theoretical Biology 243, 134 142. doi:10.1016/j.jtbi.2006.06.012

Killingback, T., Doebeli, M., Hauert, C., 2010. Diversity of cooperation in the tragedy of the commons. Biological Theory 5, 3-6. doi:10.1162/BIOT_a_00019

Killingback, T., Doebeli, M., Knowlton, N., 1999. Variable investment, the continuous prisoner's dilemma, and the origin of cooperation. Proceedings of the Royal Society of London. Series B: Biological Sciences 266, 1723 -1728. doi:10.1098/rspb.1999.0838

Leimar, O., 2009. Multidimensional convergence stability. Evolutionary Ecology Research 11, 191-208.

MaynardSmith, J., 1982. Evolution and the theory of games. Cambridge University Press. 
586

587

588

589

590

591

592

593

594

595

596

597

598

599

600

601

602

603

604

605

606

607

608

609

610

611

612
Melis, A.P., Semmann, D., 2010. How is human cooperation different? Philos Trans R Soc Lond B Biol Sci 365, 2663-2674. doi:10.1098/rstb.2010.0157

Metz, J.A.J., Geritz, S.A.H., Jacobs, F.J.A., Heerwaarden, J.S.V., 1996. Adaptive dynamics: A geometrical study of the consequences of nearly faithful reproduction, in "Stochastic and Spatial Structures of Dynamical Systems." North-Holland/Elsevier,.

Pangle, W.M., Holekamp, K.E., 2010. Functions of vigilance behaviour in a social carnivore, the spotted hyaena, Crocuta crocuta. Animal Behaviour 80, 257-267. doi:10.1016/j.anbehav.2010.04.026

Parvinen, K., 2011. Adaptive dynamics of altruistic cooperation in a metapopulation: evolutionary emergence of cooperators and defectors or evolutionary suicide? Bull. Math. Biol. 73, 2605-2626. doi:10.1007/s11538-011-9638-4

Proctor, C.J., Broom, M., Ruxton, G.D., 2001. Modelling antipredator vigilance and flight response in group foragers when warning signals are ambiguous. Journal of Theoretical Biology 211, 409-417. doi:10.1006/jtbi.2001.2353

Raihani, N.J., Thornton, A., Bshary, R., 2012. Punishment and cooperation in nature. Trends in Ecology \& Evolution 27, 288-295. doi:10.1016/j.tree.2011.12.004 Sachs, J.L., Mueller, U.G., Wilcox, T.P., Bull, J.J., 2004. The evolution of cooperation. The Quarterly Review of Biology 79, 135-160. doi:10.1086/383541

Scheuring, I., 2014. Diffusive public goods and coexistence of cooperators and cheaters on a 1D lattice. PLoS ONE 9, e100769. doi:10.1371/journal.pone.0100769

Wakano, J.Y., Iwasa, Y., 2013. Evolutionary branching in a finite population: deterministic branching vs. stochastic branching. Genetics 193, 229-241. doi:10.1534/genetics.112.144980

Zaks, A., Klibanov, A.M., 1985. Enzyme-catalyzed processes in organic solvents. PNAS 82, 3192-3196. 


\section{Figure Legend}

\section{Figure 1}

615 An image of the types of aggregation process of rewards obtained by considering

616 the combination of three aspects. In the process of the cooperation, individuals'

617 contributions are aggregated on the stage of investments (boxes) or effects (circles),

618 which eventually yields individual reward $F$. The plus and multiplication signs indicate

619 the aggregation is additive and multiplicative, respectively. The difference that the

620 reward type is promotion of advantage (arrows with plus signs) or suppression of

621 disadvantage (arrows with minus signs) also makes difference in the multiplicative

622 aggregation of effect, but in other cases it yields no difference (see main text in Model).

623 Therefore, AI, MI, and AE are represented without distinction of the difference.

624

625 Figure 2

626 An example of individual-based simulation, resulting in evolution of polymorphism

627 with more than two morphs. The darkness of the colour shows the density of the

628 individuals with the cooperation level (vertical axis) in the population at the generation

629 (horizontal axis). Under the aggregation type AI concave effect and cost functions, 630 multiple evolutionary branchings are possible. In this case, three morphs with different 631 cooperation levels emerge and coexist in the population evolutionarily. The detail of the 632 simulation and the parameter values are shown in Appendix B.

634 Figure 3

635 Relationship between branching conditions of monomorphic singular solution and 636 functional shapes of effect and cost. Rows and columns are the functional shapes of 637 effect and cost, respectively. 
639 Table Legend

640 Table 1

641 The possibilities of evolutionary and sustained polymorphism in each type of

642 aggregation process from the analytical investigation (see Appendix A).

643

\begin{tabular}{|c|c|c|c|}
\hline \multicolumn{2}{|c|}{$\begin{array}{c}\text { Type of } \\
\text { aggregation process }\end{array}$} & \multirow{2}{*}{$\begin{array}{c}\begin{array}{c}\text { Possibility of evolutionary } \\
\text { polymorphism }\end{array} \\
\text { Polymorphism }\end{array}$} & \multirow{2}{*}{$\begin{array}{c}\begin{array}{c}\text { Possibility of sustained } \\
\text { polymorphism }\end{array} \\
\text { Polymorphism }\end{array}$} \\
\hline AI & $f(\Sigma z)$ & & \\
\hline MI & $f(\Pi z)$ & Polymorphism & Polymorphism \\
\hline $\mathrm{AE}$ & $\Sigma f(\mathrm{z})$ & Monomorphism & Monomorphism \\
\hline pME & $\Pi f_{+}(z)$ & Monomorphism & Dimorphism \\
\hline sME & $-\Pi f-(z)$ & Dimorphism & Dimorphism \\
\hline
\end{tabular}

644

645 
Additive aggregati on Multiplicative aggregation of Investment (Al)

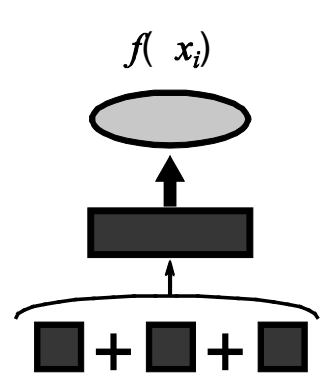

$$
\text { of Investment (MI) }
$$

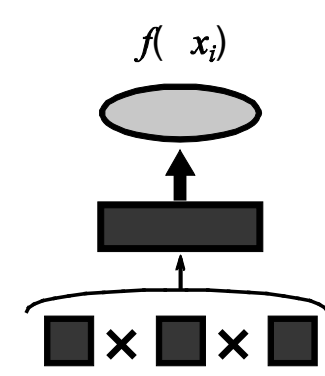

Additive aggregati on of Effect (AE)

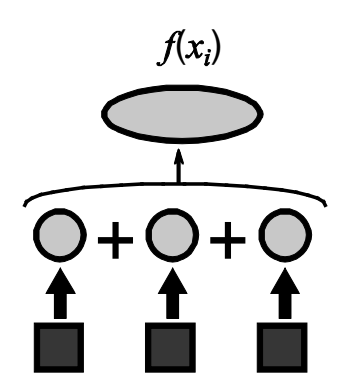

Multıplıcatıve aggregatıon Multplıc atıve Aggregatıon of Effect with Promotion of Effect with Suppressior of advantage (pME) of disadvantage (SME)

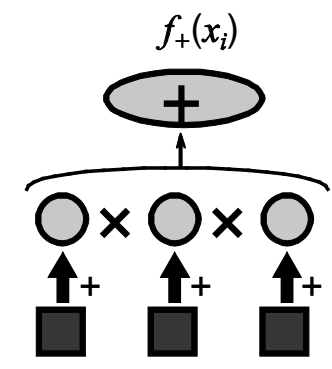

Figure 1 


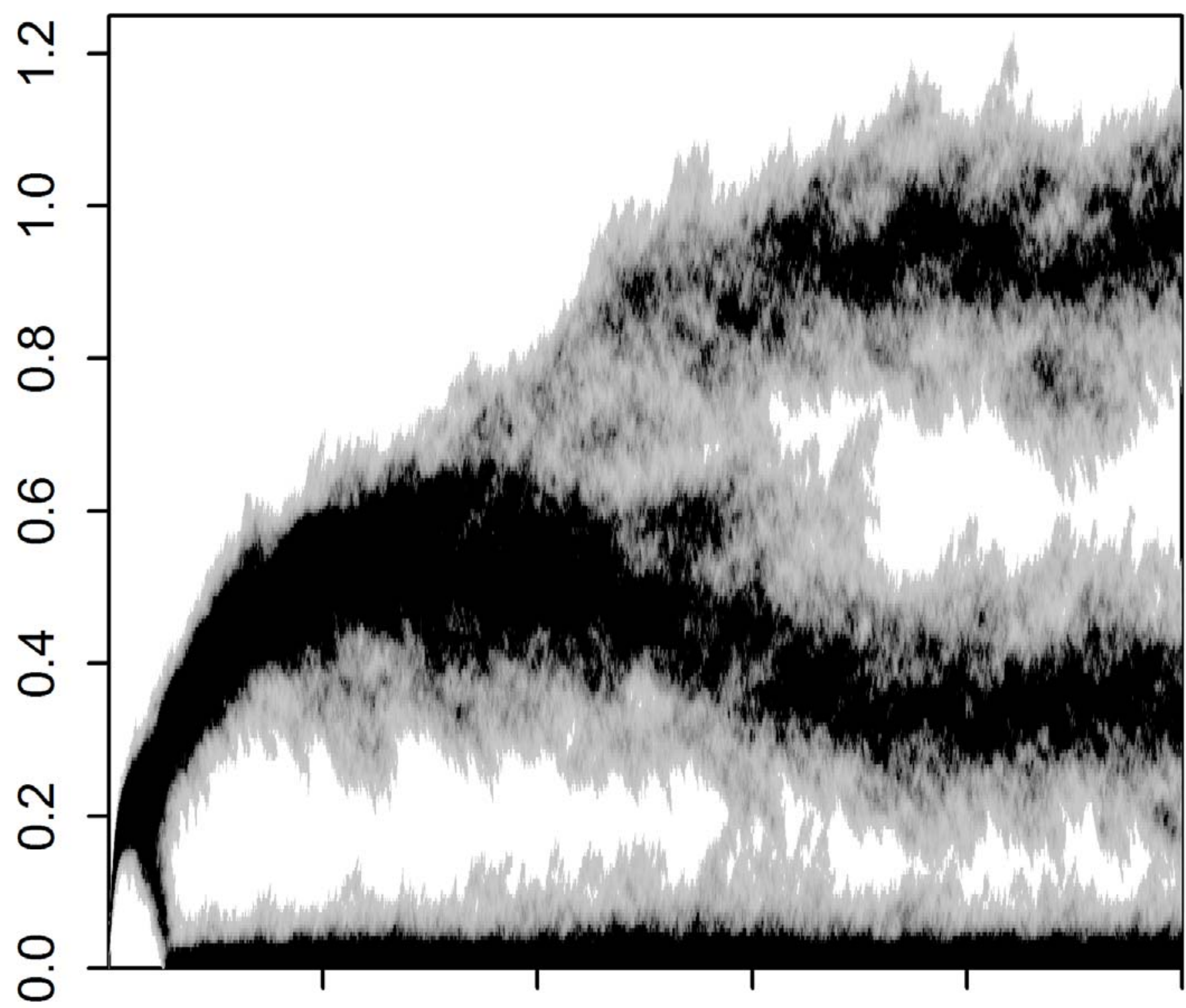

50000100000150000200000250000 


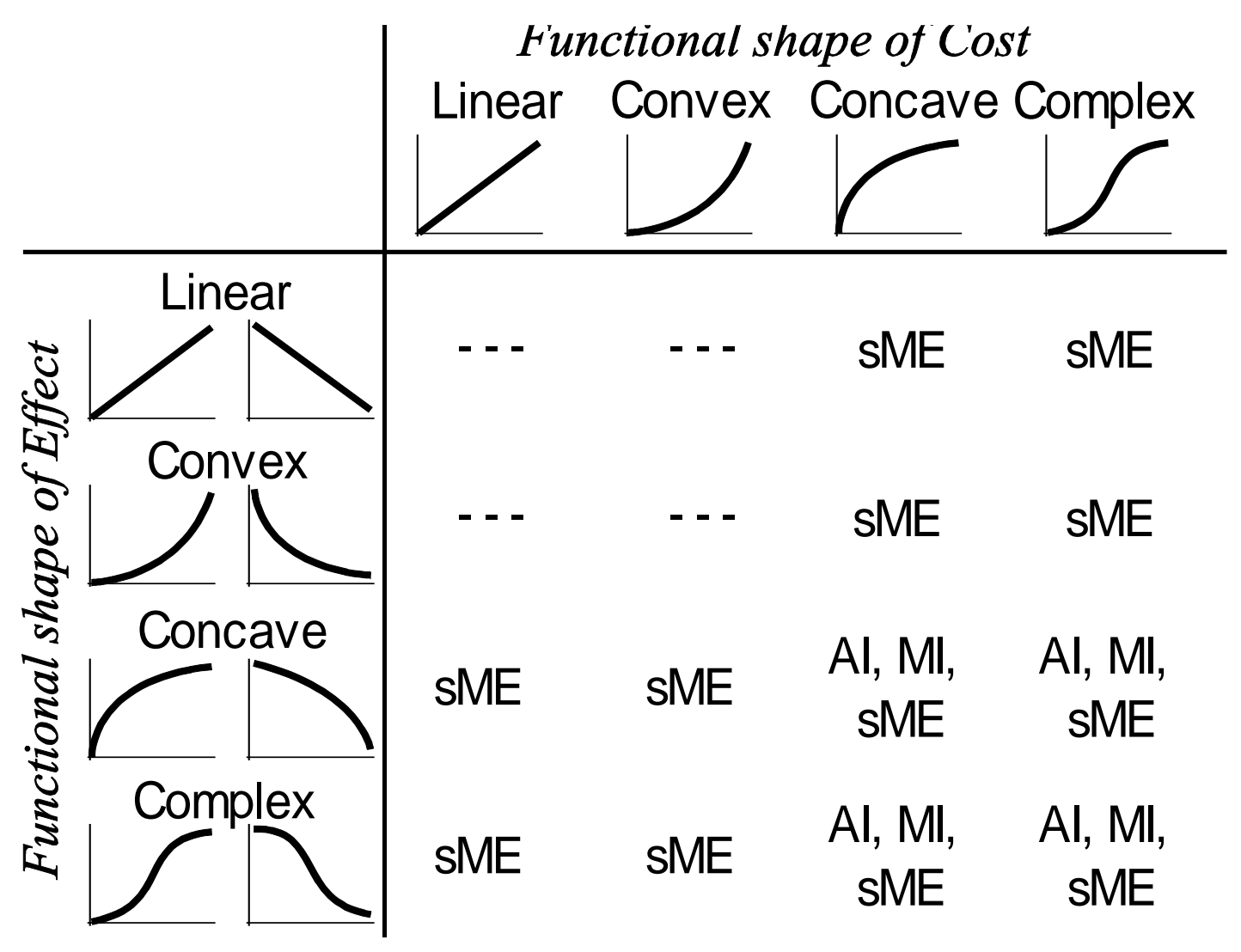

Figure 3 